\title{
Diffuse-interface model for rapid phase transformations in nonequilibrium systems
}

\author{
Peter Galenko* \\ Institut für Raumsimulation, DLR, Köln, D-51170, Germany \\ David Jou \\ Departament de Física, Universitat Autònoma de Barcelona, 08193 Bellaterra, Catalonia, Spain
}

(Received 18 August 2004; published 18 April 2005)

\begin{abstract}
A thermodynamic approach to rapid phase transformations within a diffuse interface in a binary system is developed. Assuming an extended set of independent thermodynamic variables formed by the union of the classic set of slow variables and the space of fast variables, we introduce finiteness of the heat and solute diffusive propagation at the finite speed of the interface advancing. To describe transformations within the diffuse interface, we use the phase-field model which allows us to follow steep but smooth changes of phase within the width of the diffuse interface. Governing equations of the phase-field model are derived for the hyperbolic model, a model with memory, and a model of nonlinear evolution of transformation within the diffuse interface. The consistency of the model is proved by the verification of the validity of the condition of positive entropy production and by outcomes of the fluctuation-dissipation theorem. A comparison with existing sharp-interface and diffuse-interface versions of the model is given.
\end{abstract}

DOI: 10.1103/PhysRevE.71.046125

\section{INTRODUCTION}

A classic free-boundary problem introduces, within the context of phase transformations theory, the model of phase interface with a zero thickness. Within the scope of this problem, a sharp discontinuity in the properties (or a jump of fluxes and thermodynamic functions) occurs across the interface. The sharp-interface model has been successfully used to describe many physical phenomena in various systems [1]. However, the sharp-interface model has difficulties in describing situations when the interfacial thickness becomes comparable with the characteristic length of the considered phenomenon and when a topology of the interface becomes complicated or multiply connected. To surmount these difficulties, an alternative model with a finite interfacial thickness was suggested for explaining phase transformations [2].

Historically, the first formulation of basic principles of diffuse interfaces was given by Poisson, Maxwell, and Gibbs [3] who suggested an interface be considered a region with a finite thickness in which a steep but smooth transition of physical properties of phases occurs. Lord Rayleigh, van der Waals, and Korteweg [4] applied thermodynamical principles to develop the gradient theories for interfaces with a nonzero thickness. Through the past century, these ideas of diffuse interface were refined and applied in many physical phenomena (see, e.g., overviews in Ref. [5]).

Diffuse-interface formalism has been widely applied to phase transformations in condensed media. The first introduction of the diffuse interface into the theory of phase transformations was made by Landau and Khalatnikov [6] by borrowing a formalism of the Landau theory of phase transitions [7]. Landau and Khalatnikov labeled different phases by an additional order parameter to describe anomalous

\footnotetext{
*E mail address: Peter.Galenko@dlr.de
}

sound absorption of liquid helium. In its well-known form, a formal variational approach was established by Ginzburg and Landau for the phase transitions from the normal to the superconducting phase [8]. On the basis of this approach, diffuse-interface models with order parameters have been developed by Halperin, Hohenberg, and Ma [9]. They apply these models to the theory of critical phenomena. In addition, Allen and Cahn apply the same models to antiphase domain coarsening [10].

A diffuse-interface model has also been developed for a description of phase transformations of the first order, especially for the solidification phenomenon. The diffuseinterface model of solidification incorporates an order parameter in the form of a phase-field variable [11]. The phasefield $\Phi$ has a constant value in homogeneous phases, e.g., $\Phi=-1$ for an unstable liquid phase. This phase is transformed into the solid phase with $\Phi=+1$. Between these phases in the interfacial region, the phase field $\Phi$ changes steeply but smoothly from -1 to +1 . Numerical solutions allow one to avoid explicit tracking of the interface and to locate the interface at $\Phi=0$ [12]. As a particular case, the phase-field model is reduced to sharp interface limits [13] and is consistent with major models of sharp interface (such as Hele-Shaw type models, classical or modified Stefan problem, etc.). The phase field $\Phi$ is considered as an order parameter which is introduced to describe a moving interfacial boundary between an initially unstable phase and the final phase.

Several thermodynamically consistent phase-field models have been proposed [14-17]. These include the models for a transformation in a pure system [14] as well as rather general models of multiphase transformations in multicomponent systems [17]. All of these models assume local equilibrium in a system, consistent with the basic hypothesis of classic irreversible thermodynamics (CITs) $[18,19]$. This assumption leads to an examination of a number of transport processes with small and moderate deviations from thermodynamic 
equilibrium. As a consequence, a relatively slow movement of the interface can be predicted. In principle, such an approach can be extended to a case characterized with a local violation of the condition of equilibrium at the interface, such as for solute trapping and kinetic effects [20]. However, local equilibrium is missing both at the interface and within bulk phases for cases of rapid transformation, such as rapid solidification [21]. In this case, the description of rapid phase transformations can be provided by a formalism of extended irreversible thermodynamics (EITs) [22]. This formalism gives a causal description of transport processes and abandons the assumption of local equilibrium. An extension of the phase-field methodology for a rapid transformation, which is caused by a significant deviation from a thermodynamic equilibrium, has been made recently [23].

The main purpose of the present paper is to describe a thermodynamically consistent model of rapid phase transformation in a binary system under local nonequilibrium conditions. Using the phase-field methodology, we derive governing equations compatible with the macroscopic formalism of EIT and the microscopic fluctuation-dissipation theorem.

The paper is organized as follows. In Sec. II, a thermodynamic description of the considered system is given. We introduce dissipative diffusion fluxes for heat and mass transport together with the phase-field rate of change, both as independent variables. In Sec. III, the generalized Gibbs equation and an entropy balance applicable to a rapid advancing of diffuse interfaces are given. As a starting point for the present phase-field model, an entropy functional is used in Sec. IV. The analysis of the present phase-field model leads to governing equations for a hyperbolic system with dissipation. In Sec. V, a generalization of the hyperbolic phase-field model is given using flux relaxation functions, as well as a variational principle. In Sec. VI, model equations are compared with the outcomes of existing sharp-interface and diffuse-interface models. Finally, in Sec. VII we present a summary of our conclusions.

\section{DESCRIPTION OF THE SYSTEM}

\section{A. Thermodynamic variables}

Let us consider an isobaric binary system at a nonuniform temperature $T$ with no convective flow and with given concentrations of atoms $A$ and $B$. The local equilibrium hypothesis establishes that local and instantaneous correlations among properties of the system are the same as for the whole system at a global equilibrium. Describing the nonequilibrium system as an ensemble of small local volumes in an internal equilibrium, CIT is applicable to processes not too far from the equilibrium [19]. In addition to CIT, a local nonequilibrium formalism applicable to strongly nonequilibrium systems has been developed in past two decades $[22,24-26]$. As a phenomenological theory, this formalism is well-known as extended irreversible thermodynamics, EIT $[22,27]$. This formalism goes beyond the hypothesis of local equilibrium and avoids the paradox of a propagation of disturbances with an infinite speed.

A fundamental problem in attempting to describe systems out of equilibrium is to select relevant variables needed for a valid description of a nonequilibrium state. This problem has been discussed extensively in the literature (see references in the bibliographic overview [28]). A selection of the basic state space with the inclusion of dissipative fluxes is formulated in EIT [24] and tested against experimental data [29]. Accordingly, we extend the classic set of independent thermodynamic variables by the inclusion of dissipative fluxes as additional basic variables.

CIT is based on the local equilibrium hypothesis $[18,19]$ which assumes an instant relaxation of fluxes to their steadystate values and describes the ensemble of atoms within local volumes by the Gibbs-Boltzmann statistics. In the standard formalism of the diffuse-interface using CIT, the set $\{C\}$ of independent variables is assumed to consist of conserved variables, such as energy density $e(\vec{r}, t)$ and concentration $X(\vec{r}, t)=X_{B} /\left(X_{A}+X_{B}\right)$ of the $B$ component in the system, and the nonconserved phase-field $\Phi(\vec{r}, t)$ variable (where $t$ is the time and $\vec{r}$ is the position vector of a point within system). This can be expressed formally as $\{C\}=\{e, X, \Phi\}$.

The extended space of the independent variables $\mathbf{E}$ is formed by the union of the classical set $\{C\}$ and the additional space $\{F\}$ of the fluxes of heat $\vec{q}$ and solute $\vec{J}$, and also the rate of change $\partial \Phi / \partial t$ of the phase-field variable, i.e., $\{F\}=\{\vec{q}, \vec{J}, \partial \Phi / \partial t\}$. This yields

$$
\mathbf{E}=\{C\} \cup\{F\}=\{e, X, \Phi\} \cup\{\vec{q}, \vec{J}, \partial \Phi / \partial t\} .
$$

Here $\{F\}$ is the space of the fast non conserved thermodynamic variables.

There are, in fact, different possible choices of variables (fluxes in EIT, microstructural details in theories with internal variables), and the specific choice to be adopted depends on the aims of the description and on the problems to be analyzed. This does not mean that different choices of variables are incompatible. For instance, in the study of flowing polymer solutions one may select as independent variables either the viscous pressure tensor or the conformation tensor describing the average microstructure of macromolecules of the system: a Legendre transform exists. This allows one to pass from one description to the other: similar to the way it is possible to pass from a description using internal energy as independent variable to a description using absolute temperature as an independent variable in equilibrium thermodynamics [30].

Thus, our choice of fluxes as variables does not exclude other possibilities. To justify our choice, the meaning and relevance of $\vec{q}, \vec{J}$, and $\partial \Phi / \partial t$ as variables should be reconsidered on qualitative grounds. Fluxes $\vec{q}$ and $\vec{J}$ describe exchanges of heat and matter between an interface and the neighboring bulk phases. The fluxes do not follow instantaneously classical Fourier and Fick laws. It takes them some time (usually rather short) to reach the value predicted by the classical transport equations. Obviously, when the interface motion is fast enough, delay effects in the dynamics of fluxes may play a determining role. This happens, for instance, when the velocity $V$ of the interface becomes comparable or higher than $l / \tau(l$ being the mean-free path of the particles and $\tau$ the relaxation time of fluxes). Thus, in these circum- 
stances, $\vec{q}$ and $\vec{J}$ behave as independent variables with their own dynamics, which has important consequences for the dynamics and stability of the interface [31,32].

The introduction of $\partial \Phi / \partial t$ as an additional independent variable is motivated by a similar, though slightly different consideration. Indeed, the space variation of $\Phi$ is related, among other factors, to the width of the interface. Thus, including $\partial \Phi / \partial t$ as an independent variable allows for a more detailed description of both internal kinetics and shape of the interface. In the same way as in Newtonian mechanics (where the initial position and velocity of a particle must be specified to determine their evolution), here we take both $\Phi$ and $\partial \Phi / \partial t$ as independent variables. If inertial effects are sufficiently low in comparison with dissipative effects, $\partial \Phi / \partial t$ will be determined directly by a dynamical equation in terms of $\Phi$ and its gradient. Otherwise, $\Phi$ and $\partial \Phi / \partial t$ will be independent and an equation for $\partial^{2} \Phi / \partial t^{2}$ must be found.

The above discussed choice of variables leads to two sets of independent variables as follows. Variables from the set $\{C\}$ are characterized as the slow variables. Their behavior is governed by conservation laws for energy and solute concentration plus an evolution of the phase field. They decay slowly in time. In contrast, the independent space $\{F\}$ consists of nonconserved variables with a relatively high rate of decay. The variables from $\{F\}$ differ from their classical values during the time intervals of the order of magnitude of characteristic times $\tau_{i}$ for the relaxation of the heat flux, solute diffusion flux, and rate of change of the phase-field variable, respectively. For time intervals much longer than the relaxation times $\tau_{i}$, the rate of variation of the fluxes can be ignored.

\section{B. Relaxation times}

Generally, relaxation times $\tau_{i}$ represent physically reasonable time estimations for a spontaneous return of a system to the steady state after a sudden perturbation. Relaxation times $\tau_{T}$ and $\tau_{D}$ for the heat and solute, respectively, can be considered as times needed for smoothing of inhomogeneities of temperature and concentration, respectively, by diffusion. The time $\tau_{\Phi}$ of the relaxation for the phase-field can be evaluated from the velocity of the diffuse interface moving through the local volume with the characteristic spatial length. Consequently, the rate of decay of the heat flux $\vec{q}$, solute diffusive flux $\vec{J}$, and phase-field rate of change $\partial \Phi / \partial t$ are estimated by the following characteristic times:

$$
\tau_{T}=a / V_{T}^{2}, \tau_{D}=D / V_{D}^{2}, \tau_{\Phi}=l / V,
$$

where $a$ is the thermal diffusivity, $V_{T}$ the finite speed for heat diffusion (i.e., the speed of propagation of temperature disturbances), $D$ is the solute diffusion constant, $V_{D}$ is the finite speed for diffusion (i.e., the speed of propagation of concentration disturbances), $V$ is the velocity of the diffuse interface, and $l$ is the spatial length.

For instance, the time $\tau_{T}$ is defined by phonon-electron and phonon-phonon interactions for heat diffusion in metallic systems and it is estimated in Ref. [33] to be in the range of $10^{-13} \mathrm{~s}<\tau_{T}<10^{-11} \mathrm{~s}$. The time $\tau_{D}$ is defined by the time for diffusion jumps of particles, which varies within a wide interval of $10^{-11} \mathrm{~s}<\tau_{D}<10^{-7} \mathrm{~s}$ in a binary alloy system or inorganic solution [34]. In addition to this, the time $\tau_{\Phi}$ might be evaluated numerically from Eq. (2), assuming that the length $l=W_{0}$ (the width of the diffuse interface) and the velocity $V$ (the characteristic velocity for rapid adiabatic transformations) are known. Thus, for numeric evaluation of $\tau_{\Phi}$ in a pure system, one may accept the following expression:

$$
\tau_{\Phi}=W_{0} \chi /\left(\mu_{0} Q\right),
$$

where $Q$ is the heat of transformation, $\chi$ is the heat capacity (so that relation $Q / \chi$ is considered as the characteristic temperature for adiabatic transformation), and $\mu_{0}$ is the coefficient for atomic kinetics. Taking the values for pure nickel, e.g., $Q / \chi=418 \mathrm{~K}$ [35], $\mu_{0}=0.52 \mathrm{~m} /(\mathrm{s} \mathrm{K})$ [36], and $W_{0}=5$ $\times 10^{-9} \mathrm{~m}$, one gets $\tau_{\Phi}=2.30 \times 10^{-11} \mathrm{~s}$. This value for $\tau_{\Phi}$ agrees with the time of the diffuse-interface kinetics which might be calculated from the "thin-interface" analyses of Karma and Rappel [37], extended by Bragard et al. [38].

It is also reasonable to evaluate the relaxation time for the phase-field in a binary system using the outcomes of the phase-field model via "thin-interface" analyses presented by Karma and Rappel [37] for pure substances and by Karma [39] for isothermal solidification of dilute binary systems. Namely, for nonisothermal solidification of a binary system, Ramirez et al. [40] derived the time $\tau_{\Phi}$ for the phase-field as a function of $X$ and $\Phi$. It is described by

$$
\tau_{\Phi}=\frac{W_{0}^{2}}{\Gamma}\left(\frac{1}{\mu_{0}}+a_{1} a_{2} \frac{W_{0}}{D}\left[\frac{D Q}{a \chi}+\frac{m(1-k) X}{1+k-(1-k) \Phi}\right]\right) .
$$

For numeric evaluation, we accept the following material parameters for a $\mathrm{Cu}-\mathrm{Ni}$ metallic system: diffuse-interface width $W_{0}=1 \times 10^{-9} \mathrm{~m}$, Gibbs-Thomson coefficient $\Gamma=1.3$ $\times 10^{-7} \mathrm{~K} \mathrm{~m}$ [41], atomic kinetics coefficient $\mu_{0}$ $=0.24 \mathrm{~m} /(\mathrm{s} \mathrm{K})$ [41], constants $a_{1}=0.8839 \cdots$ and $a_{2}$ $=0.6267 \cdots$ [37], solute diffusion constant $D=3 \times 10^{-9} \mathrm{~m}^{2} / \mathrm{s}$ [41], thermal diffusivity $a=1.5 \times 10^{-5} \mathrm{~m}^{2} / \mathrm{s}$ [41], adiabatic temperature (a relation of latent heat and heat capacity) $Q / \chi=402 \mathrm{~K}$ [42], slope of the liquidus line $m$ $=4.38 \mathrm{~K} /$ at. $\%$ [42], and solute partitioning coefficient $k$ $=0.81[42]$. As a result, using Eq. (4), one gets $\tau_{\Phi}=7.92$ $\times 10^{-11} \mathrm{~s}$ for the values of $X=70$ at. $\%$ and $\Phi=0.5$.

Values for relaxation times for some pure and binary systems are summarized in Table I. It can be seen, e.g., for metals and alloys, that even though the heat speed $V_{T}$ is much larger than the solute diffusion speed $V_{D}$, relaxation times for $\vec{q}$ and $\vec{J}$ may have the same order of magnitude, i.e., $\tau_{T} \approx \tau_{D}$. Therefore, the front of the heat profile moves with a speed much higher than the front of the solute diffusive profile. However, due to the fast thermal diffusion, $a \gg D$, the relaxation of the heat flux $\vec{q}$ proceeds at approximately the same characteristic time as the relaxation for solute diffusion flux $\vec{J}$.

\section{ENTROPY APPROACH}

\section{A. Generalized Gibbs equation}

For the local nonequilibrium system described in Sec. II, we postulate an existence of a local generalized entropy den- 
TABLE I. Relaxation time for the fluxes of heat, solute diffusion, and phase field.

\begin{tabular}{lccc}
\hline \hline \multicolumn{1}{c}{ System } & \multicolumn{1}{c}{$\tau_{T}(\mathrm{~s})$} & $\tau_{D}(\mathrm{~s})$ & $\tau_{\Phi}(\mathrm{s})$ \\
\hline Carbon tetrachloride & $2.50 \times 10^{-13}$, Ref. [43] & \\
Benzene & $1.22 \times 10^{-13}$, Ref. [43] & \\
Nickel & $1.20 \times 10^{-11}$, Ref. [44] & $2.30 \times 10^{-11}$, Ref. [45] \\
Diluted alloy Ni-0.7 at. \% B & & $1.54 \times 10^{-11}$, Ref. [46] \\
Concentrated alloy Cu-30 at. \% Ni & $0.75 \times 10^{-11}$, Ref. [47] $7.92 \times 10^{-11}$, Ref. [48] \\
\hline
\end{tabular}

sity $s$. The related set of variables is the extended space $\mathbf{E}$ by Eq. (1). The generalized Gibbs equation for $s$ is described by

$$
\begin{aligned}
d s(e, X, \Phi, \vec{q}, \vec{J}, \partial \Phi / \partial t)= & d s_{e}(e, X, \Phi)+d s_{n e}(\vec{q}, \vec{J}, \partial \Phi / \partial t) \\
= & \frac{\partial s_{e}}{\partial e} d e+\frac{\partial s_{e}}{\partial X} d X+\frac{\partial s_{e}}{\partial \Phi} d \Phi+\frac{\partial s_{n e}}{\partial \vec{q}} \cdot d \vec{q} \\
& +\frac{\partial s_{n e}}{\partial \vec{J}} \cdot d \vec{J}+\frac{\partial s_{n e}}{\partial(\partial \Phi / \partial t)} d\left(\frac{\partial \Phi}{\partial t}\right) .
\end{aligned}
$$

In Eq. (5), $s_{e}$ is a local equilibrium contribution defined on the set $\{C\}$ of the classic slow variables $\{e, X, \Phi\}$, and $s_{n e}$ is the flux-dependent purely nonequilibrium part of the generalized entropy defined on the space $\{F\}$ consisting of the fluxes $\{\vec{q}, \vec{J}, \partial \Phi / \partial t\}$ as the independent fast variables.

The derivatives of the entropy density, formulated with respect to classical variables and their fluxes appearing in Eq. (5) are described by

$$
\begin{gathered}
\frac{\partial s_{e}}{\partial e}=\frac{1}{T}, \quad \frac{\partial s_{e}}{\partial X}=-\frac{\Delta \mu}{T}, \\
\frac{\partial s_{e}}{\partial \Phi}=(1-X) \frac{\partial s_{A}}{\partial \Phi}+X \frac{\partial s_{B}}{\partial \Phi}, \\
\frac{\partial s_{n e}}{\partial \vec{q}}=-\alpha_{q} \vec{q}, \quad \frac{\partial s_{n e}}{\partial \vec{J}}=-\alpha_{j} \vec{J}, \\
\frac{\partial s_{n e}}{\partial(\partial \Phi / \partial t)}=-\alpha_{\phi} \frac{\partial \Phi}{\partial t},
\end{gathered}
$$

where $\Delta \mu=\mu_{A}-\mu_{B}$ is the difference of chemical potentials for components $A$ and $B$, respectively. The entities $s_{A}$ and $s_{B}$ are the entropies for pure components $A$ and $B$, respectively. The chemical potentials and entropies of components can be chosen for every concrete system (see, e.g., Refs. [15,17]).

In Eqs. (6), the coefficients $\alpha_{i}$ are scalars which do not depend on $\vec{q}, \vec{J}$, and $\partial \Phi / \partial t$ and are assumed to be

$$
\begin{gathered}
\alpha_{q}=\left(\frac{\tau_{T}}{\kappa T^{2}}\right)_{X, \Phi}, \quad \alpha_{j}=\frac{\tau_{D}}{T D}\left(\frac{\partial(\Delta \mu)}{\partial X}\right)_{T, \Phi}, \\
\alpha_{\phi}=\left(a_{0} \frac{\tau_{\Phi} W_{0} Q}{T \mu_{0}}\right)_{T, X},
\end{gathered}
$$

where $\kappa$ is the thermal conductivity, $a_{0}$ is a dimensionless factor (dependent on the model of the diffuse interface, spe- cifically leading to the sharp-interface asymptotic limit), and $Q$ is the heat of the transformation.

After integration, the generalized Gibbs equation (5) can be written in the form

$$
\begin{aligned}
& s(e, X, \Phi, \vec{q}, \vec{J}, \partial \Phi / \partial t)=s_{e}(e, X, \Phi)+s_{n e}(\vec{q}, \vec{J}, \partial \Phi / \partial t), \\
& s_{n e}(\vec{q}, \vec{J}, \partial \Phi / \partial t)=-\frac{\alpha_{q}}{2} \vec{q} \cdot \vec{q}-\frac{\alpha_{j}}{2} \vec{J} \cdot \vec{J}-\frac{\alpha_{\phi}}{2}\left(\frac{\partial \Phi}{\partial t}\right)^{2} .
\end{aligned}
$$

Consequently, we arrive at a generalized entropy density given by an expansion around its local equilibrium value up to second order in fluxes. At the limit of infinite speeds $\left(V_{T}\right.$ $\rightarrow \infty, V_{D} \rightarrow \infty$, and $V \rightarrow \infty$ ), one gets $\tau_{T} \rightarrow 0, \tau_{D} \rightarrow 0$, and $\tau_{\Phi}$ $\rightarrow 0$. In such a case, the term $s_{n e}$ vanishes and Eq. (8) gives the entropy density $s_{e}(e, X, \Phi)$ for a local equilibrium system.

\section{B. Entropy balance}

For a system described by the extended set $\mathbf{E}$ of variables, Eq. (1), the local balance laws for the energy and concentration are given by

$$
\frac{\partial e}{\partial t}+\nabla \cdot \vec{q}=0, \quad \frac{\partial X}{\partial t}+\nabla \cdot \vec{J}=0,
$$

and the evolution of entropy density is defined by

$$
\frac{\partial s}{\partial t}+\nabla \cdot \vec{J}_{S}=\sigma_{S}
$$

A change of the total entropy $S$ in time $t$ is described by

$$
\frac{d S}{d t}=\left(\frac{d S}{d t}\right)_{\mathrm{ex}}+\left(\frac{d S}{d t}\right)_{\mathrm{in}},
$$

where

$$
\left(\frac{d S}{d t}\right)_{\mathrm{ex}}=-\int_{v} \nabla \cdot \vec{J}_{S} d v=-\int_{\omega} \vec{J}_{S} \cdot \vec{n} d \omega
$$

represents an external exchange of entropy due to the entropy flux $\vec{J}_{S}$ and

$$
\left(\frac{d S}{d t}\right)_{\text {in }}=\int_{v} \sigma_{S} d v
$$

is the internal production of entropy due to the dissipation within the system. In Eqs. (12) and (13), $\omega$ is the outer sur- 
face of the subvolume $v, \vec{n}$ is the normal vector to the surface, and $\sigma_{S}$ is the local entropy production.

\section{HYPERBOLIC PHASE-FIELD MODEL}

In this section, an important class of hyperbolic models with dissipation is considered. For involved variables we will formulate explicit evolution equations including the relaxation terms.

\section{A. An entropy functional}

Now we use an entropy functional of the following form:

$$
\begin{aligned}
S= & \int_{v}\left[s(e, X, \Phi, \vec{q}, \vec{J}, \partial \Phi / \partial t)-\frac{\varepsilon_{e}^{2}}{2}|\nabla e|^{2}-\frac{\varepsilon_{x}^{2}}{2}|\nabla X|^{2}\right. \\
& \left.-\frac{\varepsilon_{\phi}^{2}}{2}|\nabla \Phi|^{2}\right] d v .
\end{aligned}
$$

Here $\varepsilon_{e}, \varepsilon_{x}$, and $\varepsilon_{\phi}$ are constants for the energy, concentration, and phase field, respectively. In the functional (14) the gradient terms $|\nabla e|^{2},|\nabla X|^{2}$, and $|\nabla \Phi|^{2}$ are used to describe a spatial inhomogeneity within the fields according to previously formulated diffuse-interface models $[8,10,12]$. It is logical to include gradient terms in Eq. (14) (of the so-called "Ginzburg-Landau form") because, as stressed before, our interest is focused on interfaces with steep gradients. In addition, the extension (1) gives the entropy density $s$ based on the fluxes $\vec{q}, \vec{J}$, and $\partial \Phi / \partial t$ as independent variables.

To obtain an evolution of the entropy, (11) and to consider several parts of the entropy transfer (12) and (13), we differentiate Eq. (14) with respect to time. Combining the terms, after some algebra one obtains

$$
\begin{aligned}
\frac{d S}{d t}= & \int_{v}\left[\frac{\partial s}{\partial e}+\varepsilon_{e}^{2} \nabla^{2} e\right]\left(\frac{\partial e}{\partial t}\right) d v+\int_{v}\left[\frac{\partial s}{\partial X}+\varepsilon_{x}^{2} \nabla^{2} X\right]\left(\frac{\partial X}{\partial t}\right) d v \\
& +\int_{v}\left[\frac{\partial s}{\partial \Phi}+\varepsilon_{\phi}^{2} \nabla^{2} \Phi\right]\left(\frac{\partial \Phi}{\partial t}\right) d v+\int_{v}\left[\frac{\partial s}{\partial \vec{q}} \cdot\left(\frac{\partial \vec{q}}{\partial t}\right)\right. \\
& \left.+\frac{\partial s}{\partial \vec{J}} \cdot\left(\frac{\partial \vec{J}}{\partial t}\right)+\frac{\partial s}{\partial(\partial \Phi / \partial t)}\left(\frac{\partial^{2} \Phi}{\partial t^{2}}\right)\right] d v-\int_{\omega}\left[\varepsilon_{e}^{2}\left(\frac{\partial e}{\partial t}\right) \nabla_{n} e\right. \\
& \left.+\varepsilon_{x}^{2}\left(\frac{\partial X}{\partial t}\right) \nabla_{n} X+\varepsilon_{\phi}^{2}\left(\frac{\partial \Phi}{\partial t}\right) \nabla_{n} \Phi\right] d \omega,
\end{aligned}
$$

where $\nabla_{n}$ is the gradient vector in the direction of the normal vector $\vec{n}$.

Now we substitute the balance laws for energy and concentration, Eqs. (9), into Eq. (15), and then use the theorem of divergence. One gets

$$
\begin{aligned}
\frac{d S}{d t}= & -\int_{\omega}\left\{\varepsilon_{e}^{2}\left(\frac{\partial e}{\partial t}\right) \nabla_{n} e+\left(\frac{\partial s}{\partial e}+\varepsilon_{e}^{2} \nabla^{2} e\right) q_{n}+\varepsilon_{x}^{2}\left(\frac{\partial X}{\partial t}\right) \nabla_{n} X\right. \\
& \left.+\left(\frac{\partial s}{\partial X}+\varepsilon_{x}^{2} \nabla^{2} X\right) J_{n}+\varepsilon_{\phi}^{2}\left(\frac{\partial \Phi}{\partial t}\right) \nabla_{n} \Phi\right\} d \omega \\
& +\int_{v}\left\{\vec{q} \cdot \nabla\left[\frac{\partial s}{\partial e}+\varepsilon_{e}^{2} \nabla^{2} e\right]+\frac{\partial s}{\partial \vec{q}} \cdot \frac{\partial \vec{q}}{\partial t}\right.
\end{aligned}
$$

$$
\begin{aligned}
& +\vec{J} \nabla\left[\frac{\partial s}{\partial X}+\varepsilon_{x}^{2} \nabla^{2} X\right]+\frac{\partial s}{\partial \vec{J}} \cdot \frac{\partial \vec{J}}{\partial t}+\frac{\partial \Phi}{\partial t}\left[\frac{\partial s}{\partial \Phi}+\varepsilon_{\phi}^{2} \nabla^{2} \Phi\right] \\
& \left.+\frac{\partial s}{\partial(\partial \Phi / \partial t)} \frac{\partial^{2} \Phi}{\partial t^{2}}\right\} d v
\end{aligned}
$$

where $q_{n}$ and $J_{n}$ are diffusion fluxes in the direction of the normal vector $\vec{n}$.

Using Eq. (6), the change of the entropy, Eqs. (11)-(13), can be obtained from Eq. (16). This yields

$$
\frac{d S}{d t}=-\int_{\omega} J_{S} d \omega+\int_{v} \sigma_{S} d v
$$

where

$$
\begin{aligned}
J_{S}= & \varepsilon_{e}^{2}\left(\frac{\partial e}{\partial t}\right) \nabla_{n} e+\left(\frac{\partial s}{\partial e}+\varepsilon_{e}^{2} \nabla^{2} e\right) q_{n}+\varepsilon_{x}^{2}\left(\frac{\partial X}{\partial t}\right) \nabla_{n} X \\
& +\left(\frac{\partial s}{\partial X}+\varepsilon_{x}^{2} \nabla^{2} X\right) J_{n}+\varepsilon_{\phi}^{2}\left(\frac{\partial \Phi}{\partial t}\right) \nabla_{n} \Phi
\end{aligned}
$$

is the projection of the entropy flux vector on the normal vector $\vec{n}$ and

$$
\begin{aligned}
\sigma_{S}= & \vec{q} \cdot\left[\nabla\left(\frac{\partial s}{\partial e}+\varepsilon_{e}^{2} \nabla^{2} e\right)-\alpha_{q} \frac{\partial \vec{q}}{\partial t}\right]+\vec{J} \cdot\left[\nabla\left(\frac{\partial s}{\partial X}+\varepsilon_{x}^{2} \nabla^{2} X\right)\right. \\
& \left.-\alpha_{j} \frac{\partial \vec{J}}{\partial t}\right]+\frac{\partial \Phi}{\partial t}\left[\frac{\partial s}{\partial \Phi}+\varepsilon_{\phi}^{2} \nabla^{2} \Phi-\alpha_{\phi} \frac{\partial^{2} \Phi}{\partial t^{2}}\right]>0
\end{aligned}
$$

is the local entropy production which has a bilinear form in terms of fluxes $(\vec{q}, \vec{J}$, and $\partial \Phi / \partial t)$ and their respective conjugate forces (the expressions inside the square brackets).

\section{B. Governing equations and thermodynamic consistency}

Relation (18) is well known from the phase-field model that is based on CIT (see, e.g., Ref. [15]), whereas the entropy production (19) includes additional terms $-\alpha_{q} \partial \vec{q} / \partial t$, $-\alpha_{j} \partial \vec{J} / \partial t$, and $-\alpha_{\phi} \partial^{2} \Phi / \partial t^{2}$ related to the nonequilibrium part of the generalized entropy. This structure is due to a special form for entropy, Eq. (8), and has a clear physical meaning: far from equilibrium, dissipative fluxes provide ordering that leads to a decrease of entropy production near a steady state when compared with the local-equilibrium state characterized by same values of $e, X$, and $\Phi$.

As a consequence of the second law of thermodynamics the production $\sigma_{S}$ of the generalized entropy (19) is positive. This condition implies a relation between fluxes and forces which, in the simplest cases, is assumed to be linear. For Eq. (19), the following set of equations can be formulated.

Evolution equations for heat and solute diffusion fluxes 


$$
\left\{\begin{array}{l}
\vec{q} \\
\vec{J}
\end{array}\right\}=(\mathcal{M})\left\{\begin{array}{c}
\nabla\left(\frac{\partial s}{\partial e}+\varepsilon_{e}^{2} \nabla^{2} e\right)-\alpha_{q} \frac{\partial \vec{q}}{\partial t} \\
\nabla\left(\frac{\partial s}{\partial X}+\varepsilon_{x}^{2} \nabla^{2} X\right)-\alpha_{j} \frac{\partial \vec{J}}{\partial t}
\end{array}\right\}
$$

and evolution equation for the phase field

$$
\frac{\partial \Phi}{\partial t}=M_{\phi}\left(\frac{\partial s}{\partial \Phi}+\varepsilon_{\phi}^{2} \nabla^{2} \Phi-\alpha_{\phi} \frac{\partial^{2} \Phi}{\partial t^{2}}\right)
$$

where

$$
(\mathcal{M})=\left(\begin{array}{ll}
M_{e e} & M_{e x} \\
M_{x e} & M_{x x}
\end{array}\right)
$$

is the matrix of mobilities for thermal and solutal transport and $M_{\phi}$ is the mobility of the diffuse interface. The interface mobility is assumed to be dependent on composition as

$$
M_{\phi}=(1-X) M_{\phi}^{A}+X M_{\phi}^{B}>0,
$$

where $M_{\phi}^{A}$ and $M_{\phi}^{B}$ are the interface mobility for the transformation in pure systems consisting of $A$ or $B$ components, respectively. In various formulations of the phase-field model $[20,37]$, the mobilities of $M_{\phi}^{A}$ and $M_{\phi}^{B}$ are proportional to the atomic interface kinetic coefficient $\mu_{0}$ and inversely proportional to the interface width $W_{0}$, so that $M_{\phi} \sim \mu_{0} / W_{0}$.

The matrix (22) of both transport and the interface mobility (23) is assumed to be positively defined for positive entropy production $\sigma_{S}$. The matrix (22) can be considered as symmetric, so that it can be regarded as being positive, i.e., $M_{e e} M_{x x}>M_{e x}^{2}$. Note that linear phenomenological laws implied by Eqs. (20) and (21) assume validity of the representation theorem of isotropic tensors [49]. According to this theorem, the fluxes and forces of different tensorial rank do not couple as long as linear relations are involved (an independence of processes of different tensorial rank known as the Curie principle). In our case, vectors of heat and solute diffusion fluxes cannot lead to the flux of the scalar phase field in a linear description. More complicated nonlinear relations between fluxes and forces, consistent with positive entropy production in EIT, are considered elsewhere $[22,24,27]$.

For simplicity, we ignore both kinds of "cross coupling" effects in Eq. (20), so that $M_{e x}=M_{x e}=0$. Then, a substitution of the fluxes from Eq. (20) into the balances (9) gives the governing equation for energy density

$$
\tau_{T} \frac{\partial^{2} e}{\partial t^{2}}+\frac{\partial e}{\partial t}=-\nabla \cdot\left[M_{e e} \nabla\left(\frac{\partial s}{\partial e}+\varepsilon_{e}^{2} \nabla^{2} e\right)\right]
$$

and the governing equation for solute concentration

$$
\tau_{D} \frac{\partial^{2} X}{\partial t^{2}}+\frac{\partial X}{\partial t}=-\nabla \cdot\left[M_{x x} \nabla\left(\frac{\partial s}{\partial X}+\varepsilon_{x}^{2} \nabla^{2} X\right)\right],
$$

in which $\tau_{T}=\alpha_{q} M_{e e}$ is the relaxation time for the heat diffusion flux, and $\tau_{D}=\alpha_{j} M_{x x}$ is the relaxation time for solute diffusion [see Eqs. (2) and Table I]. After simplifying, Eq. (21) leads to the governing equation for the phase field

$$
\tau_{\Phi} \frac{\partial^{2} \Phi}{\partial t^{2}}+\frac{\partial \Phi}{\partial t}=M_{\phi}\left(\frac{\partial s}{\partial \Phi}+\varepsilon_{\phi}^{2} \nabla^{2} \Phi\right)
$$

where $\tau_{\Phi}=\alpha_{\phi} M_{\phi}$ is the time scale of the phase-field kinetics. According to Eq. (26), the acceleration $\partial^{2} \Phi / \partial t^{2}$ of the phase field appears due to the introduction of both $\Phi$ and $\partial \Phi / \partial t$ as independent variables. The acceleration characterizes inertial effects inside the width of diffuse interface.

The derived equations (24)-(26)are the central outcome of this study [or, to mention a more complicated setting, we could also refer to equations (20) and (21)]. One role of the relaxation times is clear: they characterize the delay with which $\vec{q}$ and $\vec{J}$ reduce to their classical forms (which correspond to the classical transport equations). Furthermore, this delay indicates a loss of inertial effects in the dynamics of the interfacial region. Relaxation terms may be neglected in many circumstances, but become crucial in some important situations. For instance, they lead to a maximum possible value for the speed of advance of the interface (in contrast to the classic theory which allows for an infinite speed of propagation). Moreover, they lead to the possibility of oscillatory phenomena appearing within the domain of the interface. Thus, the role of new terms is not simply to add some new undetermined parameters (i.e., the relaxation times), allowing for an improved fit with experimental results. These terms play an important conceptual role, because they open the possibility for a drastic change in behavior of the modeled system.

Some comments on the consistency of the proposal formulated in this study can be outlined. First of all, we may refer to its internal consistency as a thermodynamic (macroscopic) theory. Second, one must check its consistency with respect to microscopic descriptions based, for instance, on kinetic theory, linear response theory, or other statistical (microscopic) theories. Furthermore, one must check its consistency with experimental results.

Here, we comment on the internal thermodynamic consistency first. In the next section, we refer to its consistency with respect to a statistical theory that is based on the fluctuation-dissipation theorem. In this theoretical paper, we do not refer to experimental results. We assume that a consistent nonequilibrium thermodynamic theory should satisfy two main conditions: (i) generalized or extended entropy must be at a maximum in the equilibrium state, (ii) entropy production must be positive. To these two conditions, one could add two more requirements: (iii) the second differential of the entropy with respect to its basic variables (which is related to the dynamics of the variables) must be negative in order to lead to dynamically stable solutions and (iv) the generalized equations of state obtained by a differentiation of the generalized entropy must have a physical meaning that would be consistent with experiments.

It can be seen immediately that the essential conditions (i) and (ii) are satisfied in our proposal. Indeed, the form (8) and (14) of the entropy guarantees that the homogeneous equilibrium state has the maximum entropy as compared to nonequilibrium states with the same local values of $e, X$, and $\Phi$. Furthermore, an introduction of constitutive equations (20) 
and (21) into the expression (19) of the entropy production yields a definite positive expression

$$
\sigma_{S}=(\vec{q}, \vec{J})(\mathcal{M})^{-1}\left\{\begin{array}{l}
\vec{q} \\
\vec{J}
\end{array}\right\}+M_{\phi}^{-1}\left(\frac{\partial \Phi}{\partial t}\right)^{2}>0 .
$$

As we noted earlier, the transport matrix $(\mathcal{M})$ and the interface mobility $M_{\phi}$ are assumed to be positively defined for a positive entropy production $\sigma_{S}>0$. If one includes higherorder nonlinear terms into the entropy (8) or in the constitutive equations (20) and (21), thermodynamic consistency would be more difficult to verify than in our second-order approximation (8). This approximation though is sufficient to deal with a wide range of physical problems.

We shall not deal with conditions (iii) and (iv), which are more subtle and typically involve nonlinear effects. For an indication of an approach to their analysis in some situations involving only $\vec{q}$ as nonequilibrium variables, the reader is referred to the monograph [24].

\section{GENERALIZATION OF THE MODEL}

The governing equations (24) and (26) present a causal propagation of heat and mass signals and indicate a dissipative-wave advance of a diffuse interface. We generalize these equations into the so-called evolution equations which are nonlinear in time. First, the equations of state are considered from the point of view of the relaxation functions for the fluxes. Second, the nonlinear evolution equations of a general type are derived from the variational formulation.

\section{A. Relaxation functions for the fluxes}

Let us take into consideration a prehistory of the change of the phase field at a point of a system. Such a prehistory must be taken into account if the system is not in a local equilibrium. We shall use a functional description with a memory function.

We use the entropy functional (14), as before, to derive the equations of the model. In the absence of local equilibrium, one may incorporate the prehistory of the diffusion process. Subsequently, connections between the fluxes $\vec{q}, \vec{J}$ and $\partial \Phi / \partial t$ from the one side and driving forces $\nabla(\delta S / \delta e)$, $\nabla(\delta S / \delta X)$, and $\delta S / \delta \Phi$, from the other side, are defined by the following integral forms. Relaxation of the heat flux

$$
\vec{q}(\vec{r}, t)=\int_{-\infty}^{t} D_{q}\left(t-t^{*}\right) \nabla \frac{\delta S\left(t^{*}, \vec{r}\right)}{\delta e} d t^{*},
$$

relaxation of the solute diffusion flux

$$
\vec{J}(\vec{r}, t)=\int_{-\infty}^{t} D_{j}\left(t-t^{*}\right) \nabla \frac{\delta S\left(t^{*}, \vec{r}\right)}{\delta X} d t^{*},
$$

and relaxation of the phase-field rate of change

$$
\frac{1}{M_{\phi}} \frac{\partial \Phi(\vec{r}, t)}{\partial t}=-\int_{-\infty}^{t} D_{\phi}\left(t-t^{*}\right) \frac{\delta S\left(t^{*}, \vec{r}\right)}{\delta \Phi} d t^{*},
$$

where $D_{R}=\left\{D_{q}, D_{j}, D_{\phi}\right\}$ are the relaxational kernels for the fluxes and the variational derivatives are obtained from the following expressions:

$$
\begin{gathered}
\frac{\delta S}{\delta e}=\frac{\partial s}{\partial e}+\varepsilon_{e}^{2} \nabla^{2} e, \quad \frac{\delta S}{\delta X}=\frac{\partial s}{\partial X}+\varepsilon_{x}^{2} \nabla^{2} X, \\
\frac{\delta S}{\delta \Phi}=\frac{\partial s}{\partial \Phi}+\varepsilon_{\phi}^{2} \nabla^{2} \Phi .
\end{gathered}
$$

After substitution of expressions for the heat flux relaxation (28) and the solute diffusion relaxation (29), into the balance laws for energy and solute concentration (9), respectively, one can obtain the following integro-differential equations:

$$
\begin{aligned}
& \frac{\partial e(\vec{r}, t)}{\partial t}=-\nabla \cdot \int_{-\infty}^{t} D_{q}\left(t-t^{*}\right) \nabla \frac{\delta S\left(t^{*}, \vec{r}\right)}{\delta e} d t^{*}, \\
& \frac{\partial X(\vec{r}, t)}{\partial t}=-\nabla \cdot \int_{-\infty}^{t} D_{j}\left(t-t^{*}\right) \nabla \frac{\delta S\left(t^{*}, \vec{r}\right)}{\delta X} d t^{*} .
\end{aligned}
$$

Together with relaxation of the phase field (30), the general system evolution during the phase transformation is described by Eqs. (32).

When the relaxation functions $D_{R}$ are specially defined, Eqs. (30) and (32) can be reduced to known models. Specifically, for an important class of dissipative and hyperbolic models, one can take the relaxation kernels in the following forms:

$$
D_{R}= \begin{cases}D_{R}(0) \equiv \text { const }, & \text { wave propagation, } \\ D_{R}(0) \delta\left(t-t^{*}\right), & \text { dissipation, } \\ D_{R}(0) \exp \left(-\frac{t-t^{*}}{\tau_{i}}\right), & \text { wave and dissipation, }\end{cases}
$$

where $D_{R}(0)=\left\{D_{q}(0), D_{j}(0), D_{\phi}(0)\right\}$ are the relaxational kernels for the fluxes at the present time $t=t^{*}$, and $\tau_{i}$ $=\left\{\tau_{T}, \tau_{D}, \tau_{\Phi}\right\}$ are the characteristic relaxation times for the fluxes.

Different transformations within a diffuse interface are described by different kernels in the integrals (28)-(30). As it follows from Eq. (33), the relaxation functions $D_{R}$ describe the memory of the system by assigning different weights to different moments in the past. The dissipation corresponds to a zero-memory transformation, i.e., the only relevant contributions are the "last"ones. In contrast to this situation, the infinite memory transformation with $D_{R} \equiv$ const leads to an undamped wave propagation of the heat, solute, or the interface advancement. In between, the combination of the wave and dissipative regimes described by the exponentional law can be observed during rapid phase transformations. This is the case of a hyperbolic phase-field model described in Sec. 
IV. For the latter case, the relevance of all contributions to the fluxes decreases as the system moves to the past.

In Sec. IV, the macroscopic consistency of the statements of EIT has been shown. Now, the consistency of our macroscopic approach is verified with microscopic description. It is related to the consequences obtained from the fluctuationdissipation theorem.

The memory functions introduced in Eqs. (28)-(30) may be related to our analysis of the dynamics of the fluxes $\vec{q}$ and $\vec{J}$ and of $\partial \Phi / \partial t$ proposed by constitutive equations (20) and (21). To do this, first, we may consider the fluctuationdissipation theorem by relating the response memory functions to the time-correlation function of the corresponding fluxes (see, e.g., Ref. [50]). This will allow us to show the consistency of our macroscopic formulation with the microscopic basis provided by the fluctuation-dissipation theorem.

The corresponding expressions are

$$
\begin{gathered}
D_{q}\left(t-t^{*}\right)=\frac{1}{k_{B} T^{2}}\left\langle\hat{\vec{q}}(t) \hat{\vec{q}}\left(t^{*}\right)\right\rangle_{\mathrm{eq}}, \\
D_{j}\left(t-t^{*}\right)=\frac{1}{k_{B} T}\left\langle\hat{\vec{J}}(t) \hat{\vec{J}}\left(t^{*}\right)\right\rangle_{\mathrm{eq}}, \\
D_{\phi}\left(t-t^{*}\right)=\frac{1}{k_{B} T}\left\langle\partial_{t} \hat{\Phi}(t) \partial_{t} \hat{\Phi}\left(t^{*}\right)\right\rangle_{\mathrm{eq}} .
\end{gathered}
$$

Here $k_{B}$ is Boltzmann's constant; $\vec{q}, \vec{J}$, and $\partial_{t} \Phi$ stand for the microscopic operators for the heat flux, diffusion flux and the time derivative of $\Phi$, respectively; and $\langle\ldots\rangle_{\text {eq }}$ means an average over an equilibrium ensemble in statistical mechanics (as, for instance, the canonical one).

Relations (34) play an important role in modern statistical mechanics, and may be formally derived from the Liouville equation in the framework of the linear-response theory or from information theory $[50,51]$. However, from a practical point of view, a computation of the evolution of microscopic operators for $\vec{q}, \vec{J}$ or $\partial_{t} \Phi$ on purely microscopic grounds is an overwhelming task that exceeds the actual capabilities of an analyst. Such an evolution is either obtained by computer simulations, or inferred on heuristic grounds. Thus, our evolution equations (20) and (21) for $\vec{q}, \vec{J}$, and $\partial_{t} \Phi$ may be considered within the scope of macroscopic modeling of the evolution of fluxes, which according to Eq. (34) is equivalent to a proposal form of corresponding memory functions introduced in Eqs. (28)-(30). In general terms, it could be said that, according to Eq. (34), the study of the evolution of the fluxes in the neighborhood of the equilibrium is equivalent to the determination of the corresponding memory functions.

Constitutive equations (20) and (21) imply that fluctuations of $\vec{q}$ and $\vec{J}$ near a homogeneous equilibrium state will decay exponentially as $\vec{q}(t)=\vec{q}(0) \exp \left(-t / \tau_{T}\right)$ and $\vec{J}(t)$ $=\vec{J}(0) \exp \left(-t / \tau_{D}\right)$. Introducing these expressions into Eq. (34) one obtains

$$
D_{q}\left(t-t^{*}\right)=\frac{1}{k_{B} T^{2}}\langle\hat{\vec{q}}(0) \hat{\vec{q}}(0)\rangle_{\mathrm{eq}} \exp \left(-\frac{t-t^{*}}{\tau_{T}}\right),
$$

$$
D_{j}\left(t-t^{*}\right)=\frac{1}{k_{B} T}\langle\hat{\vec{J}}(0) \hat{\vec{J}}(0)\rangle_{\mathrm{eq}} \exp \left(-\frac{t-t^{*}}{\tau_{D}}\right),
$$

which may be rewritten as

$$
\begin{aligned}
& D_{q}\left(t-t^{*}\right)=D_{q}(0) \exp \left(-\frac{t-t^{*}}{\tau_{T}}\right), \\
& D_{j}\left(t-t^{*}\right)=D_{j}(0) \exp \left(-\frac{t-t^{*}}{\tau_{D}}\right) .
\end{aligned}
$$

Indeed, when the microscopic expressions for $\vec{q}$ and $\vec{J}$ (corresponding to an ideal gas) are introduced into Eq. (36) and an equilibrium averaging is performed over a MaxwellBoltzmann distribution function, the results for the $D_{q}(0)$ and $D_{j}(0)$ became equivalent to those obtained from the kinetic theory of gases in the time-relaxation approximation [50].

Note, finally, that the transport coefficients (thermal conductivity, diffusion coefficient) may be obtained (when the relaxation time is sufficiently short) by integration of Eq. (34), as

$$
\begin{aligned}
& \lambda=\frac{1}{k_{B} T^{2}} \int_{-\infty}^{\infty}\langle\hat{\vec{q}}(t) \hat{\vec{q}}(0)\rangle_{\mathrm{eq}} d t, \\
& D=\frac{1}{k_{B} T} \int_{-\infty}^{\infty}\langle\hat{\vec{J}}(t) \hat{\vec{J}}(0)\rangle_{\mathrm{eq}} d t,
\end{aligned}
$$

which are the well-known Green-Kubo formulas for transport coefficients $[24,50,51]$. Thus, our macroscopic formalism is consistent with the microscopic fluctuation-dissipation theorem. It provides, in fact, a phenomenological complement to fluctuation-dissipation expressions, which are the formal expressions. Note that it is difficult to obtain on exact grounds the form of the memory functions from these formal expressions.

\section{B. A variational principle and Euler-Lagrange equations}

We assume, as above, that the generalized entropy density $s$ is a continuous and differentiable function defined by the local equilibrium contribution $s_{e}$ and the flux-dependent nonequilibrium part $s_{\mathrm{ne}}$ with the total set of variables (1) and the generalized Gibbs equation (5). The balance equations for the heat and solute are the same, Eqs. (9), and the local evolution of the entropy density is described by Eqs. (10).

A generalization can be formulated by introducing generalized terms for derivatives of the entropy density with respect to classical variables $(e, X, \Phi)$ and fluxes $(\vec{q}, \vec{J}, \partial \Phi / \partial t)$, as well as by introducing the general forms of the entropy flux $\vec{J}_{S}$ and the source $\sigma_{S}$ in Eq. (10). Depending on their own tensorial character, these are

$$
\begin{gathered}
\left(\frac{\partial s}{\partial e}\right)_{\vec{q}}=\beta_{1}^{e}\left(e, I_{q}\right), \quad\left(\frac{\partial s}{\partial X}\right)_{\vec{J}}=\beta_{1}^{X}\left(X, I_{j}\right), \\
\left(\frac{\partial s}{\partial \Phi}\right)_{\partial \Phi / \partial t}=\beta_{1}^{\Phi}\left(\Phi, I_{\partial \Phi / \partial t}\right), \quad\left(\frac{\partial s}{\partial \vec{q}}\right)_{e}=\beta_{2}^{e}\left(e, I_{q}\right) \vec{q},
\end{gathered}
$$




$$
\begin{gathered}
\left(\frac{\partial s}{\partial \vec{J}}\right)_{X}=\beta_{2}^{X}\left(X, I_{j}\right) \vec{J}, \\
\left(\frac{\partial s}{\partial(\partial \Phi / \partial t)}\right)_{\Phi}=\beta_{2}^{\Phi}\left(\Phi, I_{\partial \Phi / \partial t}\right) \frac{\partial \Phi}{\partial t}, \\
\vec{J}_{S}=\beta_{3}^{e}\left(e, I_{q}\right) \vec{q}+\beta_{3}^{X}\left(X, I_{j}\right) \vec{J}+\beta_{3}^{\Phi}\left(\Phi, I_{\partial \Phi / \partial t}\right) \frac{\partial \Phi}{\partial t}, \\
\sigma_{S}=\beta_{4}^{e}\left(e, I_{q}\right)+\beta_{4}^{X}\left(X, I_{j}\right)+\beta_{4}^{\Phi}\left(\Phi, I_{\partial \Phi / \partial t}\right),
\end{gathered}
$$

where

$$
I_{q}=\vec{q} \cdot \vec{q}, \quad I_{j}=\vec{J} \cdot \vec{J}, \quad I_{\partial \Phi / \partial t}=\left(\frac{\partial \Phi}{\partial t}\right)^{2}
$$

are the single scalar invariants of the extended set (1) of variables, and $\beta_{i}$ are the scalar functions depending on classic variables $(e, X, \Phi)$ and invariants $I_{i}$. Then, utilizing Eqs. (38), the generalized Gibbs equation (5) gives the time derivative of the entropy density as follows:

$$
\begin{aligned}
\frac{\partial s}{\partial t}= & \beta_{1}^{e}\left(e, I_{q}\right) \frac{\partial e}{\partial t}+\beta_{2}^{e}\left(e, I_{q}\right) \vec{q} \cdot \frac{\partial \vec{q}}{\partial t}+\beta_{1}^{X}\left(X, I_{j}\right) \frac{\partial X}{\partial t} \\
& +\beta_{2}^{X}\left(X, I_{j}\right) \vec{J} \cdot \frac{\partial \vec{J}}{\partial t}+\beta_{1}^{\Phi}\left(\Phi, I_{\partial \Phi / \partial t}\right) \frac{\partial \Phi}{\partial t} \\
& +\beta_{2}^{\Phi}\left(\Phi, I_{\partial \Phi / \partial t}\right) \frac{\partial \Phi}{\partial t} \frac{\partial^{2} \Phi}{\partial t^{2}} .
\end{aligned}
$$

Locally, Eq. (10) is satisfied as a balance law. For the entire system, one can postulate an extremal condition in the Lagrangian form $\mathcal{L}=\int_{v}\left(\partial s / \partial t+\nabla \cdot \vec{J}_{S}-\sigma_{S}\right) d v \rightarrow$ extr, implying an extremal difference between the "kinetic" part $\int_{v}\left(\partial s / \partial t+\nabla \cdot \vec{J}_{S}\right) d v$ and the "potential" part $\int_{v} \sigma_{S} d v$ for the whole nonequilibrium system. Then, the entropy density satisfies the following variational principle [52]:

$$
\delta \mathcal{L}=\delta \int_{v} d v\left(\frac{\partial s}{\partial t}+\nabla \cdot \vec{J}_{S}-\sigma_{S}\right)=0
$$

in which the variation $\delta$ is carried out only on the nonconserved flux variables $\vec{q}, \vec{J}$, and $\partial \Phi / \partial t$, i.e., $\delta$ is taken only over the space $\{F\}$ from the set (1) while the variables $e, X$, and $\Phi$ from the set $\{C\}$ remain constant during the variation. Also, during the variation, the tangent thermodynamic space [time and spatial derivatives from the set (1)] is fixed. From this it follows that Eq. (41) is a variational principle of a restricted type.

Using the balance laws (9), a substitution of Eqs. (38) into the variational principle (41) leads to

$$
\begin{aligned}
& \delta \int_{v} d v\left[\left(\beta_{3}^{e}-\beta_{1}^{e}\right) \nabla \cdot \vec{q}+\left(\beta_{2}^{e} \frac{\partial \vec{q}}{\partial t}+\nabla \beta_{3}^{e}\right) \cdot \vec{q}-\beta_{4}^{e}\right. \\
& +\left(\beta_{3}^{X}-\beta_{1}^{X}\right) \nabla \cdot \vec{J}+\left(\beta_{2}^{X} \frac{\partial \vec{J}}{\partial t}+\nabla \beta_{3}^{X}\right) \cdot \vec{J}-\beta_{4}^{X}+\beta_{1}^{\Phi} \frac{\partial \Phi}{\partial t} \\
& \left.+\beta_{3}^{\Phi} \nabla \frac{\partial \Phi}{\partial t}+\left(\beta_{2}^{\Phi} \frac{\partial^{2} \Phi}{\partial t^{2}}+\nabla \beta_{3}^{\Phi}\right) \frac{\partial \Phi}{\partial t}-\beta_{4}^{\Phi}\right]=0
\end{aligned}
$$

Variation of Eq. (42) is obtained by taking as constants the time derivatives, gradients, and divergences. Since $\delta I_{q}$ $=2 \vec{q} \cdot \delta \vec{q}, \quad \delta I_{j}=2 \vec{J} \cdot \delta \vec{J}$, and $\delta I_{\partial \Phi / \partial t}=2(\partial \Phi / \partial t) \delta(\partial \Phi / \partial t)$ from Eq. (42) one gets

$$
\begin{aligned}
& \int_{v} d v\left[2\left(\frac{\partial \beta_{3}^{e}}{\partial I_{q}}-\frac{\partial \beta_{1}^{e}}{\partial I_{q}}\right) \vec{q}(\nabla \cdot \vec{q})+\beta_{2}^{e} \frac{\partial \vec{q}}{\partial t}+2 \frac{\partial \beta_{2}^{e}}{\partial I_{q}} \vec{q} \vec{q} \cdot \frac{\partial \vec{q}}{\partial t}+\nabla \beta_{3}^{e}\right. \\
& \left.-2 \frac{\partial \beta_{4}^{e}}{\partial I_{q}} \vec{q}\right] \delta \vec{q}+\int_{v} d v\left[2\left(\frac{\partial \beta_{3}^{X}}{\partial I_{j}}-\frac{\partial \beta_{1}^{X}}{\partial I_{j}}\right) \vec{J}(\nabla \cdot \vec{J})+\beta_{2}^{X} \frac{\partial \vec{J}}{\partial t}\right. \\
& \left.+2 \frac{\partial \beta_{2}^{X}}{\partial I_{j}} \vec{J} \vec{J} \cdot \frac{\partial \vec{J}}{\partial t}+\nabla \beta_{3}^{X}-2 \frac{\partial \beta_{4}^{X}}{\partial I_{j}} \vec{J}\right] \delta \vec{J} \\
& \quad+\int_{v} d v\left[2 \frac{\partial \beta_{1}^{\Phi}}{\partial I_{\partial \Phi / \partial t}}\left(\frac{\partial \Phi}{\partial t}\right)^{2}+2 \frac{\partial \beta_{3}^{\Phi}}{\partial I_{\partial \Phi / \partial t}} \frac{\partial \Phi}{\partial t} \nabla \frac{\partial \Phi}{\partial t}+\beta_{2}^{\Phi} \frac{\partial^{2} \Phi}{\partial t^{2}}\right. \\
& \left.\quad+2 \frac{\partial \beta_{2}^{\Phi}}{\partial I_{\partial \Phi / \partial t}} \frac{\partial^{2} \Phi}{\partial t^{2}}\left(\frac{\partial \Phi}{\partial t}\right)^{2}+\nabla \beta_{3}^{\Phi}-2 \frac{\partial \beta_{4}^{\Phi}}{\partial I_{\partial \Phi / \partial t}} \frac{\partial \Phi}{\partial t}\right] \delta\left(\frac{\partial \Phi}{\partial t}\right) \\
& =0 .
\end{aligned}
$$

Due to arbitrary variation of $\delta \vec{q}, \delta \vec{J}$, and $\delta(\partial \Phi / \partial t)$, the EulerLagrange equations directly follow from Eq. (43). These are evolution equation for the heat flux

$$
\begin{aligned}
\left(\frac{\partial \beta_{2}^{e}}{\partial I_{q}}\right. & \left.\vec{q} \vec{q}+\beta_{2}^{e} \mathcal{U}\right) \cdot \frac{\partial \vec{q}}{\partial t}+\left[\left(\frac{\partial \beta_{3}^{e}}{\partial I_{q}}-\frac{\partial \beta_{1}^{e}}{\partial I_{q}}\right) \nabla \cdot \vec{q}-\frac{\partial \beta_{4}^{e}}{\partial I_{q}}\right] \vec{q} \\
& =-\frac{1}{2} \nabla \beta_{3}^{e},
\end{aligned}
$$

evolution equation for the solute diffusion flux

$$
\begin{aligned}
& \left(\frac{\partial \beta_{2}^{X}}{\partial I_{j}} \vec{J} \vec{J}+\beta_{2}^{X} \mathcal{U}\right) \cdot \frac{\partial \vec{J}}{\partial t}+\left[\left(\frac{\partial \beta_{3}^{X}}{\partial I_{j}}-\frac{\partial \beta_{1}^{X}}{\partial I_{j}}\right) \nabla \cdot \vec{J}-\frac{\partial \beta_{4}^{X}}{\partial I_{j}}\right] \vec{J} \\
& =-\frac{1}{2} \nabla \beta_{3}^{X},
\end{aligned}
$$

and evolution equation for the phase-field

$$
\begin{gathered}
{\left[\frac{\partial \beta_{2}^{\Phi}}{\partial I_{\partial \Phi / \partial t}}\left(\frac{\partial \Phi}{\partial t}\right)^{2}+\frac{1}{2} \beta_{2}^{\Phi}\right] \frac{\partial^{2} \Phi}{\partial t^{2}}+\left[\frac{\partial \beta_{3}^{\Phi}}{\partial I_{\partial \Phi / \partial t}} \nabla \frac{\partial \Phi}{\partial t}+\frac{\partial \beta_{1}^{\Phi}}{\partial I_{\partial \Phi / \partial t}} \frac{\partial \Phi}{\partial t}\right.} \\
\left.-\frac{\partial \beta_{4}^{\Phi}}{\partial I_{\partial \Phi / \partial t}}\right] \frac{\partial \Phi}{\partial t}=-\frac{1}{2} \nabla \beta_{3}^{\Phi}
\end{gathered}
$$

where $\mathcal{U}$ is the unit tensor of second rank.

Equations (44)-(46) are the nonlinear evolution equations for $\vec{q}, \vec{J}$, and $\partial \Phi / \partial t$ and they are of the general form of evolution equations (20) and (21). Indeed, the nonlinearity is 
clearly seen from the following form of these equations:

$$
\begin{gathered}
\tau_{T}(e, \vec{q}) \frac{\partial \vec{q}}{\partial t}+\vec{q}=M_{e e}(e, \vec{q}) \nabla \beta_{3}^{e}, \\
\tau_{D}(X, \vec{J}) \frac{\partial \vec{J}}{\partial t}+\vec{J}=M_{x x}(X, \vec{J}) \nabla \beta_{3}^{X}, \\
\tau_{\Phi}\left(\Phi, \frac{\partial \Phi}{\partial t}\right) \frac{\partial^{2} \Phi}{\partial t^{2}}+\frac{\partial \Phi}{\partial t}=M_{\Phi}\left(\Phi, \frac{\partial \Phi}{\partial t}\right) \nabla \beta_{3}^{\Phi},
\end{gathered}
$$

where $\tau_{i}$ and $M_{i}$ are the functions of the classic set $\{C\}$ $=\{e, X, \Phi\}$ as well as nonlinear functions of the fluxes which can be found explicitly from Eqs. (44)-(46) and relations (38) and (39). Thus, taking the generalized evolution of the entropy density (40) and using variational principle (41), we arrive to nonlinear general evolution equations for fluxes (47), which can be reduced to evolution equations (20) and (21) of the hyperbolic phase-field model.

\section{RELATION TO EXISTING MODELS}

It is interesting to note that sharp-interface and diffuseinterface models with relaxation of fluxes have been used to describe transient processes in various nonequilibrium systems (see Ref. [53], Chap. 4). Therefore we synthesize here several previous and some very recent results in comparison with the developed here hyperbolic model (Sec. IV) and the generalized model (Sec. V) of rapid phase transformation.

\section{A. Superconductivity}

Ginzburg and Landau established their variational principle for a continuous transition from the normal to the superconducting phase [8]. They used a free energy density with a gradient term which has been used in further studies of many phenomena (e.g., in a description of the spinodal decomposition [54] or crystal growth [55]). As a logical extension, transition between the normal and superconducting phases can be described with a delay imposed by equations of the hyperbolic model [starting from the functional of the form (14)] or using generalized models with a memory, Sec. V A.

Generally, Eqs. (24)-(26) are consistent with the generalized entropy density given by Eq. (8). The equations are reduced to the classic equations from Refs. $[8,54,55]$ when the times tend to zero. Furthermore, the entropy density (8) together with the evolution equations (20) has been justified microscopically $[24,26]$ for the one-component system and from Grad's procedure for monatomic gases.

The choice of thermodynamic potential is important, as it governs the transition from a metastable state to the stable one. Normally, the potential for transition is included in the expression for the entropy density (or for the free energy density) in a form of a double-well function, or by a monotonically increasing function incorporating nonequilibrium conditions at the interface $[8,12,38]$. In the present paper, we do not give an explicit form of $s_{e}$ in Eq. (8) and present governing equations (24)-(26) [or variational derivatives
(31)] in a general form. However, the choice of a thermodynamic potential might be given for a problem under consideration.

\section{B. Glass transition, structural relaxation, and phase separation}

Jäckle et al. [56] considered isothermal phase transformation in the presence of additional slow structural relaxation variables. Considering the dynamics based on the relaxational chemical potential, these authors refer their model to systems with phase separation and to slow structural relaxation in polymeric solutions in proximity to the glass transition temperature. Calculations have shown that, even at the early stages of phase separation, the equation for chemical potential with a memory may give pronounced deviations from the predictions obtained by the classic Cahn-Hillard model [54].

Phase separation during spinodal decomposition may proceed under local nonequilibrium conditions in a solute diffusion field during rapid quenching. As it has been demonstrated in computational modeling [57], rapidly quenched liquid mixtures under a decomposition exhibit nonequilibrium patterns, evolving with universalities different from those extracted from the Cahn and Hillard model.

Local nonequilibrium separation in liquids can be described in terms of EIT. In these cases, such description leads to a model for isothermal spinodal decomposition in a binary system [23] (under conditions of large deviations from the thermodynamic equilibrium). The dynamics of the diffusion flux $\vec{J}$ [as a fast variable from the set (1)] is consistent with the characteristic time of the local rearrangement of particles (atoms or molecules), or with the time of relaxation of the diffusive flux to its local equilibrium steady-state value. The model equation for spinodal decomposition of a binary system is the generalized Cahn-Hillard equation (25). It is in the form of local nonequilibrium solute redistribution. In this case, the dynamics of rapidly quenched decomposition is described for short periods of time, or for large gradients of chemical composition.

\section{Shear flow, viscoelastic fluids, and diffusion-reaction systems}

The system of coupled evolution equations (20) and (21) describes, in fact, a process of the phase separation under shear if temperature is replaced by a viscous pressure tensor. In this case, one may get the required condition by defining the spinodal line in nonequilibrium states [see Ref. [30], Chap. 6]. In reduced form, an equation of type (20) or (32), accompanied with homogeneous Dirichlet boundary conditions, has been introduced to model behavior of certain viscoelastic fluids as well as to predict the velocity of flow [58].

In addition, an equation of type (32) is used to predict the wave front in time-delayed reaction-diffusion systems of the generalized Fisher's equation [59]. The speed of the traveling wave depends on the relaxation time, and therefore spreading of the population in a reaction-diffusion system can be predicted with great flexibility. One of the consequences of this 
equation, reduced to equations Eqs. (24) or (25) for modeling hyperbolic reaction-diffusion systems [with $\varepsilon_{e}=0$ or $\varepsilon_{x}=0$, respectively], can be considered in an exciting example suggested by Fort and Mendez in Ref. [60] for advance of Neolithic human groups across Europe. They have shown that hyperbolic reaction-diffusion equations of type of Eqs. (24) or (25) predict population spreading during the European past, in agreement with existing archaeological data.

\section{Rapid solidification}

At deep supercoolings in a solidifying system, or at high velocities of the solid-liquid interface, it is necessary to take into account local nonequilibrium effects in solute diffusion phenomena and to use a non-Fickian model for transport processes compatible with EIT [21,41]. The problem of rapid solidification within the sharp-interface limit is described by the generalized Stefan problem (the so-called "self-consistent hyperbolic Stefan problem" [31,32]). The analysis in such cases takes into account local nonequilibrium both at the interface and within the bulk phases. Consequently, the spatiotemporal evolution of solute concentration is described by the partial differential equation (25) of a hyperbolic type (with $\varepsilon_{x}=0$ ) which takes into account the relaxation of solute diffusion flux into the local thermodynamical equilibrium in a rapidly solidifying system.

The phenomenon of an advancement of diffuse-interfaces with higher velocities comparable with the solute diffusion speed can also be described by the phase-field model with a relaxation of the diffusion flux [23]. It has been shown that by choosing the concrete form of entropy (as the thermodynamic potential), one may recover the existing models based on the CIT and analyze solidification under local nonequilibrium conditions.

\section{E. Motion of antiphase domains}

In the description of diffuse interface kinetics, Allen and Cahn [10] proposed a model for describing the evolution of a nonconserved order field during the antiphase domain coarsening. For isotropic interfaces, the gradient flow leads to the Allen-Cahn equation by taking $\tau_{\Phi}=0$ in Eq. (26). This equation is valid in cases of low inertial effects versus dissipative effects. With a finite relaxation time $\tau_{\Phi}$ and a finite acceleration $\partial^{2} \Phi / \partial t^{2}$, Eq. (26) predicts evolution of coarsening with relaxation. It is reasonable to say that the generalized AllenCahn equation (26) is valid for cases of significant inertial effects during motion of antiphase domains.

A modification of the Allen-Cahn model formulated for the process of the interface motion by mean curvature with a delayed response has been analyzed recently. Rotstein et al. [61] developed the phase-field model based on equations similar to Eqs. (28) and (30). These authors described the first-order transition with the delayed response of the system under conditions of a slow relaxation of internal variables. Using the exponential relaxation function for a wave and dissipative mode [which leads to the hyperbolic phase-field model (33)] the dynamics of a perturbed motion of interfaces by a mean curvature has been considered. It has been shown in Ref. [61] that the internal relaxation effects induce damped oscillations in an interfacial motion during the crystalline coarsening. As opposed to the classic parabolic phasefield model, the hyperbolic phase-field model predicts these interfacial oscillations in qualitative consistency with the oscillations on the surface of quantum crystals [62], as is the case with crystallization waves in helium [63]. From a mathematical viewpoint, a search for the existence and uniqueness of the solution for some well-posed problems of a motion by mean curvature (using the phase-field model with memory) is in progress (see Ref. [64]).

\section{F. Complex (dusty) plasmas}

Recent investigations within the field of complex (dusty) plasma physics show that this system exhibits complicated behavior which depends on the behavior of its "subsystems." These subsystems are represented by electrons, ions, neutral gas, and charged dust particles. All of them have their own relaxation times to reach the local equilibrium; therefore, interactions among them may lead to a delay of relaxation to the local equilibrium in a plasma. Moreover, in the electronic subsystem of a plasma, local equilibrium does not exist. Such situations stimulate development of theories beyond a local equilibrium [65]. Interactions among different subsystems in complex (dusty) plasmas with a missing local thermodynamic equilibrium in the electronic subsystems make description of observed experimental data of this system rather complicated.

Experimental results of Morfill et al. [66] related to plasma observations show unusual behavior attributed to weak collisionless interactions of gases inherent to fluid flow with further possible crystallization of plasma. These results are described by means of molecular dynamic simulations [66]. The field approach seems also to be applicable due to the fact that during transitions in plasma, the characteristic size of patterns is on the mesoscopic or even macroscopic scale. The field approach to a heat- and electronicallyconducting fluids has been demonstrated in ionized gases [52] by using the generalized type of Eqs. (44)-(46).

\section{CONCLUSIONS}

The diffuse-interface model for rapid phase transformations in metastable binary systems has been presented. To describe the steep but smooth change of phases within the width of a diffuse interface, the formalism of the phase-field model has been used.

It is emphasized that a rapid phase transformation may proceed under local nonequilibrium conditions. In a phenomenological macroscopic description, we extend the classic set of independent thermodynamic variables by an inclusion of dissipative fluxes as additional basic variables. Evolution of the fluxes is characterized by their own dynamics with relaxation times $\tau_{i}$ summarized in Table I. Thus, the extended set (1) of variables allows one to describe phase transformations with finite interface velocity comparable or even higher than $l / \tau$, where $l$ is the mean-free-path of particles (atoms).

Evolution equations for the hyperbolic phase-field model with dissipation are derived from entropy functional (14) 
based on the extended set (1) of independent thermodynamic variables. This model yields a definite, positive entropy production (27), in full agreement with the second law of thermodynamics.

A generalization of the model has been formulated by introducing the memory functions and using a variational principle. As a result, consistency of the macroscopic approach with the microscopic fluctuation-dissipation theorem has been found for the phase-field with memory [Eqs. (34)-(37)]. Nonlinear evolution equations (44)-(46) are derived from the variational principle (41).

Derived equations for an evolution of diffuse interface were correlated with existing models of nonequilibrium transport processes and for the systems experiencing phase transformations. Particularly, we compare our derivation with the models of superconductivity, phase separation, vis- coelastic or electronically conducting fluids, interface motion by mean curvature, rapidly solidifying systems, and reactiondiffusion systems.

\section{ACKNOWLEDGMENTS}

P.G. acknowledges financial support from the German Research Foundation [DFG (Deutsche Forschungsgemeinschaft)] under the Project No. HE 1601/13. He also acknowledges the support of the Administration of the Physical Statistics Group during his stay in Universitat Autónoma de Barcelona. D.J. acknowledges financial support from the Dirección General de Investigación of the Spanish Ministry of Science and Technology Grant No. BFM 2003-06033 and the Direcció General de Recerca of the Generalitat of Catalonia under Grant No. 2001 SGR-00186.
[1] Moving Boundary Problems in Heat Flow and Diffusion, edited by J. R. Ockendon and W. R. Hodgkins (Oxford University Press, Oxford, 1975); Moving Boundary Problems, edited by D. G. Wilson, A. D. Solomon, and P. T. Boggs, (Academic Press, New York, 1978); A. Friedman, Variational Principles and Free-Boundary Problems (Wiley, New York, 1982).

[2] G. Caginalp, Arch. Ration. Mech. Anal. 92, 205 (1986).

[3] S. D. Poisson, Nouvelle Théorie de l'Action Capillaire (Bachelier, Paris, 1831); J. C. Maxwell, in Encyclopaedia Britannica, 9th ed. (1876). The Scientific Papers of James Clerk Maxwell (New York, Dover, 1952) Vol. 2, p. 541; J. W. Gibbs, The Scientific Papers of J. Willard Gibbs (Longmans, Green, London 1906), p. 55.

[4] Lord Rayleigh, Philos. Mag. 33, 209 (1892); J. D. van der Waals, J. Stat. Phys. 20, 179 (1979), translation from the original work of 1893; D. J. Korteweg, Arch. Néerl. Sci. Exactes Nat. Ser. II 6, 1 (1901).

[5] H. E. Stanley, Introduction to Phase Transitions and Critical Phenomena (Oxford University Press, Oxford, 1971); J. S. Rowlinson and B. Widom, Molecular Theory of Capillarity (Clarendon, Oxford, 1989).

[6] L. D. Landau and I. M. Khalatnikov, Dokl. Akad. Nauk SSSR 96, 469 (1954); Collected Papers of L. D. Landau, edited by D. ter Haar (Pergamon Press, Oxford, 1965), p. 626.

[7] L. D. Landau, JETP 7, 19 (1937); Collected Papers of L. D. Landau [6], p. 193.

[8] V. L. Ginzburg and L. D. Landau, JETP 20, 1064 (1950); Collected Papers of L. D. Landau [6], p. 546.

[9] B. I. Halperin, P. C. Hohenberg, and S.-K. Ma, Phys. Rev. B 10, 139 (1974).

[10] S. E. Allen and J. W. Cahn, Acta Metall. 27, 1085 (1979).

[11] G. J. Fix, in Free Boundary Problems: Theory and Applications, edited by A. Fasano and M. Primicerio (Pitman, Boston, 1983), p. 580; J. B. Collins and H. Levine, Phys. Rev. B 31, 6119 (1985); J. S. Langer, in Directions in Condensed Matter Physics, edited by G. Grinstein and G. Mazenko (World Scientific, Philadelphia, 1986) p. 165.

[12] L. Q. Chen, Annu. Rev. Mater. Res. 32, 113 (2002); W. J. Boettinger, J. A. Warren, C. Beckermann, and A. Karma, ibid.
32, 163 (2002).

[13] G. Caginalp, Phys. Rev. A 39, 5887 (1989); G. Caginalp and E. A. Socolovsky, J. Comput. Phys. 95, 85 (1991).

[14] O. Penrose and P. C. Fife, Physica D 43, 44 (1990).

[15] Z. Bi and R. F. Sekerka, Physica A 261, 95 (1998).

[16] D. M. Anderson, G. B. McFadden, and A. A. Wheeler, Physica D 135, 175 (2000).

[17] H. Garcke, B. Nestler, and B. Stinner, SIAM J. Appl. Math. 64, 775 (2004).

[18] L. Onsager, Phys. Rev. 37, 495 (1931); I. Progogine, Introduction to Thermodynamics of Irreversible Process (Interscience, New York, 1967).

[19] S. De Groot and P. Mazur, Non-equilibrium Thermodynamics (North-Holland, Amsterdam, 1962); P. Glansdorff and I. Prigogine, Thermodynamic Theory of Structure, Stability and Fluctuations (Wiley, New York, 1971).

[20] A. A. Wheeler, W. J. Boettinger, and G. B. McFadden, Phys. Rev. E 47, 1893 (1993); S. L. Wang and R. F. Sekerka, ibid. 53, 3760 (1996); W. J. Boettinger and J. A. Warren, J. Cryst. Growth 200, 583 (1999).

[21] P. Galenko and S. Sobolev, Phys. Rev. E 55, 343 (1997); P. Galenko, Phys. Rev. B 65, 144103 (2002).

[22] D. Jou, J. Casas-Vazquez, and G. Lebon, Rep. Prog. Phys. 51, 1005 (1988).

[23] P. Galenko, Phys. Lett. A 287, 190 (2001).

[24] D. Jou, J. Casas-Vazquez, and G. Lebon, Extended Irreversible Thermodynamics, 2nd ed. (Springer, Berlin, 1996).

[25] D. Joseph and L. Preziosi, Rev. Mod. Phys. 61, 41 (1989); 62, 375 (1990).

[26] I. Müller and T. Ruggeri, Extended Thermodynamics (Springer, New York, 1993).

[27] D. Jou, J. Casas-Vazquez, and G. Lebon, Rep. Prog. Phys. 62, 1035 (1999).

[28] D. Jou, J. Casas-Vazquez, and G. Lebon, J. Non-Equil. Thermodyn. 23, 277 (1998).

[29] R. Luzzi, A. R. Vasconcellos, J. Casas-Vazquez, and D. Jou, Physica A 248, 111 (1998).

[30] D. Jou, J. Casas-Vazquez, and M. Criado-Sancho, Thermodynamics of Fluids Under Flow (Springer, Berlin, 2000). 
[31] P. K. Galenko and D. A. Danilov, Phys. Lett. A 278, 129 (2000).

[32] P. K. Galenko and D. A. Danilov, J. Cryst. Growth 216, 512 (2000); Phys. Rev. E 69, 051608 (2004).

[33] R. Peierls, Quantum Theory of Solids (Oxford University Press, London, 1955).

[34] P. Galenko, Kristallografiya 38(6), 238 (1993) [Crystallogr. Rep. 38, 836 (1993)]; Phys. Lett. 190, 292 (1994).

[35] M. Barth, F. Joo, B. Wei, and D. M. Herlach, J. Non-Cryst. Solids 156-158, 398 (1993).

[36] J. J. Hoyt, B. Sadigh, M. Asta, and S. M. Foiles, Acta Mater. 47, 3181 (1999).

[37] A. Karma and W.-J. Rappel, Phys. Rev. E 57, 4323 (1998).

[38] J. Bragard, A. Karma, Y. H. Lee, and M. Plapp, Interface Sci. 10(2-3), 121 (2002).

[39] A. Karma, Phys. Rev. Lett. 87, 115701 (2001).

[40] J. C. Ramirez, C. Beckermann, A. Karma, and H.-J. Diepers, Phys. Rev. E 69, 051607 (2004).

[41] P. K. Galenko and D. A. Danilov, Phys. Lett. A 235, 271 (1997); J. Cryst. Growth 197, 992 (1999).

[42] R. Willnecker, D. M. Herlach, and B. Feuerbacher, Appl. Phys. Lett. 56, 324 (1990).

[43] R. E. Nettleton, Phys. Fluids 3, 216 (1960).

[44] Evaluated from Eq. (2) for $\tau_{T}$ in which the thermal diffusivity $a=1.2 \times 10^{-5}\left(\mathrm{~m}^{2} / \mathrm{s}\right)$ and the thermal speed $V_{T}=10^{3}(\mathrm{~m} / \mathrm{s})$ are accepted from Ref. [31].

[45] Evaluated from Eq. (3) with the material parameters given in Sec. II B.

[46] Evaluated from Eq. (2) for $\tau_{D}$ in which the diffusion constant $D=5.5 \times 10^{-9}\left(\mathrm{~m}^{2} / \mathrm{s}\right)$ and the solute diffusion speed $V_{D}$ $=18.9(\mathrm{~m} / \mathrm{s})$ are accepted from Ref. [41].

[47] Evaluated from Eq. (2) for $\tau_{D}$ in which the diffusion constant $D=3 \times 10^{-9}\left(\mathrm{~m}^{2} / \mathrm{s}\right)$ and the solute diffusion speed $V_{D}$ $=20(\mathrm{~m} / \mathrm{s})$ are accepted from Ref. [41].

[48] Evaluated from Eq. (4) with the material parameters given in Sec. II B.

[49] C. Truesdell and W. Noll, in Handbuch der Physik III, edited by S. Flugge (Springer, Berlin, 1960).
[50] P. Resibois and M. de Leener, Classical Kinetic Theory of Fluids (Wiley, New York, 1977); J. P. Hansen and I. R. McDonald, Theory of Simple Liquids (Academic, New York, 1986).

[51] D. N. Zubarev, V. Morozov, and G. Röpke, Statistical Mechanics of Nonequilibrium Processes (Akademie Verlag, Berlin, 1977), (2 volumes); R. Luzzi, A. R. Vasconcellos, and J. G. Ramos, Foundation of a Nonequilibrium Ensemble Formalism (Kluwer, Dordrecht, 2002).

[52] F. Vazquez and J. A. del Rio, Phys. Rev. E 47, 178 (1993).

[53] R. Temam, Inifinite-Dimensional Dynamical Systems in Mechanics and Physics, 2nd ed. (Springer, New York, 1997).

[54] J. W. Cahn and J. E. Hillard, J. Chem. Phys. 28, 258 (1958).

[55] J. W. Cahn, Acta Metall. 8, 554 (1960).

[56] J. Jäckle, and H. L. Frish, J. Polym. Sci., Polym. Phys. Ed. 23, 675 (1985); K. Binder, H. L. Frish, and J. Jäckle, J. Chem. Phys. 85, 1505 (1986).

[57] S. Bastea and J. L. Lebowitz, Phys. Rev. E 52, 3821 (1995).

[58] W. E. Olmstead, S. H. Davis, S. Rosenblat, and W. I. Kath, SIAM J. Appl. Math. 46, 171 (1986); B. R. Duffy, P. Freitas, and M. Grinfeld, SIAM J. Appl. Math. 33, 1090 (2002).

[59] J. Fort and V. Mendez, Rep. Prog. Phys. 65, 895 (2002).

[60] J. Fort and V. Mendez, Phys. Rev. Lett. 82, 867 (1999).

[61] H. G. Rotstein, S. Brandon, A. Novick-Cohen, and A. Nepomnyashchy, SIAM J. Appl. Math. 62, 264 (2001).

[62] A. F. Andreev and A. Y. Parshin, Sov. Phys. JETP 48, 763 (1978).

[63] A. Y. Keshishev, A. Y. Parshin, and A. V. Babkin, Sov. Phys. JETP 30, 56 (1990).

[64] A. Novick-Cohen, in Free Boundary Problems. Proceedings of the Research Institute for Mathematical Sciences. Kyoto University Meeting No. 1210 (RIMS, Kyoto, Japan, 2000) p. 129; M. Grasselli and H. G. Rotstein, J. Math. Anal. Appl. 261, 205 (2001); M. Grasselli and V. Pata, J. Evol. Equ. 4, 27 (2004).

[65] I. V. Tokatly and O. Pankratov, Phys. Rev. B 62, 2759 (2000).

[66] G. E. Morfill, S. A. Khrapak, A. V. Ivlev, B. A. Klumov, M. Rubin-Zuzic, and H. M. Thomas, Phys. Scr. T107, 59 (2004). 